\title{
Facteurs déterminants et leviers de réduction de l'usage des antibiotiques en productions animales
}

Factors affecting and measures to decrease antimicrobial use in livestock production

\section{Guillaume Lhermie, Didier Raboisson, Stéphane Krebs et Pierre Dupraz}

\section{(2) OpenEdition}

\section{Journals}

Édition électronique

URL : http://journals.openedition.org/economierurale/4671

DOI : 10.4000/economierurale.4671

ISSN : 2105-2581

Éditeur

Société Française d'Économie Rurale (SFER)

Édition imprimée

Date de publication : 30 septembre 2015

Pagination : $3-22$

ISSN : 0013-0559

Référence électronique

Guillaume Lhermie, Didier Raboisson, Stéphane Krebs et Pierre Dupraz, « Facteurs déterminants et leviers de réduction de l'usage des antibiotiques en productions animales », Économie rurale [En ligne], 348 | juillet-août 2015, mis en ligne le 01 janvier 2017, consulté le 19 avril 2019. URL : http:// journals.openedition.org/economierurale/4671; DOI : 10.4000/economierurale.4671 


\title{
Facteurs déterminants et leviers de réduction de l'usage des antibiotiques en productions animales
}

\author{
Guillaume LHERMIE • LUNAM Université, Oniris, École nationale vétérinaire, agroalimentaire \\ et de l'alimentation Nantes Atlantique, UMR Biologie, Épidémiologie et Analyse de Risque en \\ santé animale, CS 40706, Nantes; INRA, UMR1300, Nantes; Vétoquinol, Recherche et Dévelop- \\ pement, Lure \\ guillaume.lhermie@vetoquinol.com
}

Didier RABOISSON • Université de Toulouse, Institut National Polytechnique (INP), École nationale vétérinaire de Toulouse (ENVT), Toulouse ; INRA, UMR1225, Interaction hôte Agent Pathogène (IHAP), Toulouse

d.raboisson@envt.fr

Stéphane KREBS • INRA, UMR1300 Biologie, Épidémiologie et Analyse de risque en santé animale, CS 40706, Nantes; LUNAM Université, Oniris, École nationale vétérinaire, agroalimentaire et de I'alimentation Nantes Atlantique, UMR BioEpAR, Nantes

stephane.krebs@oniris-nantes.fr

Pierre DUPRAZ • INRA, UMR1302 SMART, Structures et Marchés agricoles, Ressources et Territoires, Rennes, France ; AGROCAMPUS OUEST, UMR1302 SMART, Structures et Marchés agricoles, Ressources et Territoires, Rennes, France

pierre.dupraz@rennes.inra.fr

La sélection de bactéries résistantes aux antibiotiques transmissibles à l'homme, induite par l'usage des antibiotiques en productions animales, incite les pouvoirs publics à réduire leur utilisation, dans le cadre du Plan Ecoantibio 2012-2017 et de la Loi d'avenir pour l'agriculture. Cet article questionne la pertinence des mesures en cours d'implémentation par les administrations françaises au regard d'un cadre d'analyse, regroupant dans un modèle conceptuel l'ensemble des facteurs sanitaires, économiques et institutionnels déterminant la demande d'antibiotiques. La compréhension des clés d'usage des antibiotiques, produit banal et substituable, ou au contraire ressource particulière, soumise à prescription, permet également d'envisager d'autres politiques de réduction.

MOTS-CLÉS : antibiotique, antibiorésistance, analyse économique, productions animales, politiques publiques

\section{Factors affecting and measures to decrease antimicrobial use in livestock production}

The growing concern of the selection of bacteria resistant to antimicrobials and their possible transmissions to humans lead French Authorities to implement several legislative measures to decrease antimicrobial use in livestock production. This context leads to question whether the demand of antimicrobials could be regarded as a simple demand of agricultural input, or rather as a demand of an input with distinctive effects, submitted to regulatory prescription. In this paper, the accuracy of the regulatory measures implemented by the Authorities are assessed, regarding the whole factors gathered in a conceptual framework enlightening the decision-making process of antimicrobial use. Lastly, other alternative policies are described.

(JEL : Q $12 ; Q 16 ; Q 18)$.

KEYWORDS: antimicrobials, antimicrobial resistance, economic analysis, livestock production, agricultural policy 
D écouverts dans les années 1930, les antibiotiques sont utilisés dans la lutte contre les maladies infectieuses d'origine bactérienne (Bentley et Bennett, 2003). Chez les animaux de rente, les antibiotiques sont utilisés dans trois circonstances : (i) en médecine individuelle, pour traiter un animal malade (traitement curatif) ; (ii) en médecine collective, pour traiter des lots d'animaux, lorsqu'une proportion du lot est malade (métaphylaxie) ; (iii) en prévention, avant l'apparition de la maladie, en traitant l'ensemble des animaux d'un lot pour lequel la probabilité de survenue de la maladie est considérée comme élevée (antibioprévention ou antibioprophylaxie).

En soignant certaines maladies ou en cherchant à prévenir leur survenue, les traitements antibiotiques répondent à des enjeux (i) de bien-être animal, dans le cadre de l'optimisation de la qualité des soins ; (ii) économiques, dès lors que les animaux sont élevés pour produire des denrées animales, et (iii) de santé publique, dans la lutte contre les maladies infectieuses contagieuses et particulièrement les zoonoses (maladies animales pouvant être transmises à l'homme) (Lhermie, 2010). Bien que leur intérêt médical ne soit pas remis en question, l'usage des antibiotiques comporte néanmoins des risques. Les risques directs pour la santé des animaux ou des consommateurs demeurent minimes : depuis la Loi de 1975 relative à la pharmacie vétérinaire, la mise sur le marché et la vente des médicaments sont réglementées ; la création en 1994 de l'Agence nationale du médicament vétérinaire (ANMV), intégrée en 2010 à l'Agence nationale de sécurité sanitaire des aliments de l'environnement et du travail (Anses), a pour mission d'évaluer l'efficacité et l'innocuité des médicaments avant et après leur mise sur le marché. En revanche, le recours aux antibiotiques engendre une externalité majeure, qualifiée d'usure des antibiotiques, qui s'exprime en santé animale et surtout en santé humaine (Singer et al., 2003). L'usure précoce des molécules se traduit par une inactivité progressive des antibiotiques sur les bactéries, alors dites résistantes aux antibiotiques. Certaines bactéries résistantes, sélectionnées sous la pression d'usage des antibiotiques, peuvent être disséminées chez l'homme, soit par le biais d'une proximité homme-animal, soit via la chaîne alimentaire (Angulo et al., 2004 ; Wang et al., 2012). Depuis les années 1990, des politiques publiques ont été mises en place pour atténuer ce risque : l'augmentation en médecine hospitalière de la prévalence des entérocoques résistants aux glycopeptides dans les années 1990 a ainsi conduit à l'interdiction de l'usage de l'avoparcine en médecine vétérinaire ; en 2000, le Parlement européen a interdit l'usage des antibiotiques à des doses subthérapeutiques dans l'alimentation animale comme promoteurs de croissance, réservant l'usage des antibiotiques aux seuls traitements à visées curative ou préventive (European Union, 2003). En novembre 2011, l'Union européenne s'est engagée dans un plan de maîtrise du risque de santé publique associé à l'usage des antibiotiques en médecine vétérinaire. En France, le Plan national de réduction des risques d'antibiorésistance en médecine vétérinaire (Plan Ecoantibio, 2012-2017), annoncé par le ministère de l'Agriculture en novembre 2011, vise par exemple à réduire l'usage des antibiotiques de $25 \%$ entre 2012 et 2017 (ministère de l'Agriculture, de l'Alimentation, de la Pêche et de la Ruralité, 2011). La Loi d'avenir pour l'agriculture (LAA), votée au Parlement à l'automne 2014, encadre également les pratiques en matière d'usage, de prescription et de délivrance des antibiotiques, et impose une réduction de $25 \%$ de l'usage des antibiotiques d'importance 
critique $^{1}$ entre 2013 et 2016 . Ces éléments de régulation renforcent principalement l'encadrement de l'usage des antibiotiques en productions animales, en raison du devenir de ces productions (intégrant la chaîne alimentaire humaine), et des volumes d'antibiotiques consommés par les animaux de rente. En 2005, la quantité d'antibiotiques consommée en France s'établissait ainsi à 1320 tonnes d'antibiotiques en médecine vétérinaire, contre 760 tonnes en médecine humaine (Moulin et al., 2008). Les animaux de compagnie ne représentaient que $2 \%$ du tonnage consommé en médecine vétérinaire en 2011 (Chevance et Moulin, 2012) ${ }^{2}$.

Indépendamment des aspects strictement sanitaires, les antibiotiques utilisés en élevage peuvent être vus comme des inputs contribuant à la réalisation d'un objectif économique, en œuvrant pour la «bonne » santé du troupeau et sa productivité (McInerney, 1996). Le contexte de réduction de l'usage des antibiotiques conduit à analyser les déterminants de leur demande dans les filières de production d'animaux de rente. Cet article se propose, dans une première section, de montrer en quoi la consommation d'antibiotiques en productions animales répond à un problème économique, en s'appuyant premièrement sur un cadre d' analyse couramment utilisé en économie de la santé animale, avant d'aborder les facteurs influençant leur consommation. Ce cadrage est ensuite

1. En médecine humaine, les antibiotiques d'importance critique correspondent aux classes thérapeutiques utilisées pour traiter des maladies graves sans autre alternative selon des critères définis par l'Organisation mondiale de la santé. La liste d'antibiotiques critiques encadrée par la LAA comprend aujourd'hui les fluoroquinolones et les céphalosporines de $3^{\mathrm{e}}$ et $4^{\mathrm{e}}$ générations.

2. Comme en médecine humaine, la demande d'antibiotiques pour le soin des animaux de compagnie répond très majoritairement à des objectifs strictement médicaux et ne sera pas discutée dans cet article. mobilisé dans la section suivante qui examine, de manière théorique et à la lueur d'une revue bibliographique, les leviers implémentés entre 2012 et 2014 par les politiques publiques visant à réduire l'impact de ces facteurs sur la consommation d'antibiotiques; des leviers alternatifs y sont également proposés.

\section{Les antibiotiques des inputs au service de la maîtrise des maladies}

En dégradant la santé des animaux, les maladies infectieuses altèrent quantitativement et qualitativement l'output, l'efficacité des inputs et in fine le revenu de l'éleveur. Cette section propose en premier lieu un cadre d'analyse théorique des facteurs d'apparition des maladies, de leurs impacts et de l'usage des antibiotiques pour leur contrôle. La dernière partie de cette section s'attache à préciser les autres facteurs pouvant affecter l'usage des antibiotiques, non abordés par le cadre théorique.

\section{Facteurs d'apparition exogènes et endogènes des maladies}

Les maladies infectieuses sont la conséquence de la présence simultanée d'un agent pathogène et d'un « terrain » propice à l'émergence de la maladie. La probabilité d'apparition d'une maladie dépend donc à la fois de facteurs exogènes, qui s'imposent à l'éleveur, mais aussi de facteurs endogènes, qui sont maîtrisables par l'éleveur (figure 1).

Les conditions climatiques constituent un exemple de facteurs exogènes, dans la mesure où ils influent sur la maladie, comme l'illustre par exemple la saisonnalité des troubles respiratoires d'origine infectieuse chez le jeune bovin, plus fréquents en période hivernale (Cernicchiaro et al., 2012). La prévalence régionale (qui s'apparente à un risque de contracter la maladie) constitue également un facteur 
Figure 1. Modèle conceptuel décrivant les principaux facteurs influençant les décisions individuelles de recours aux antibiotiques et les leviers de diminution

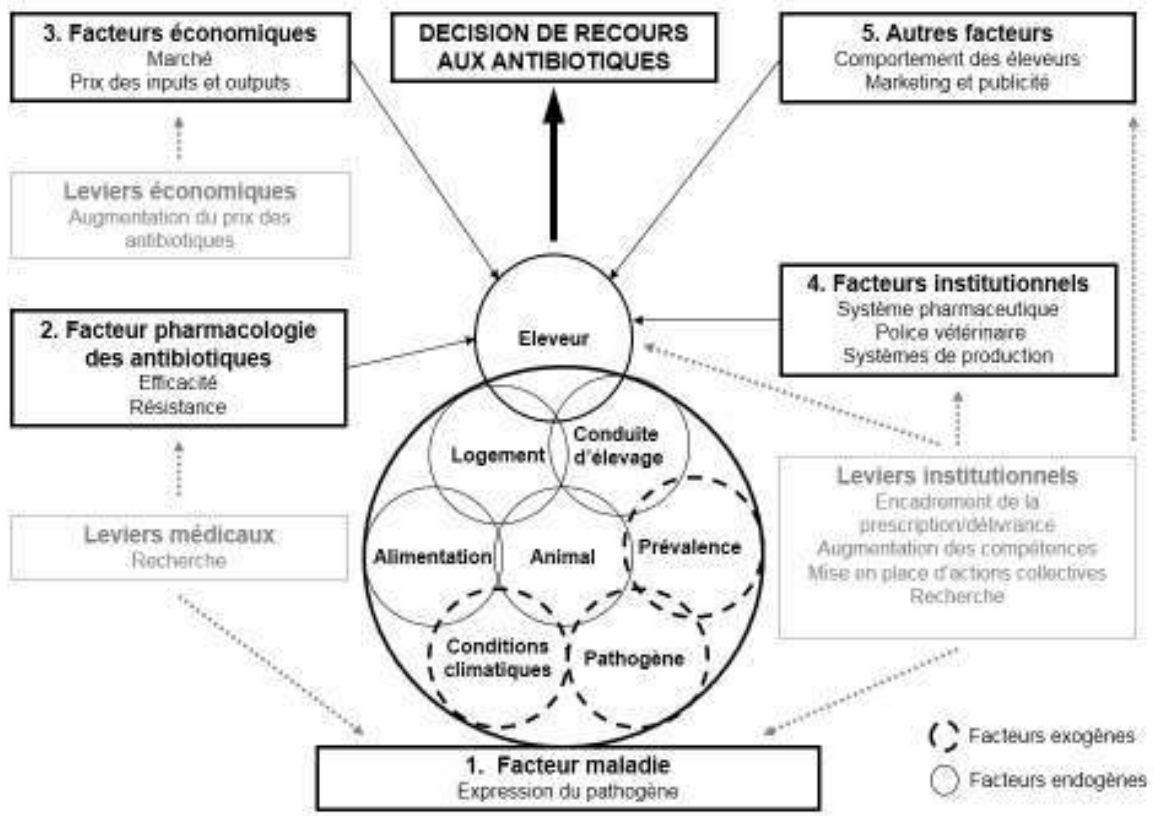

Source : les auteurs.

exogène pour une exploitation. Elle est susceptible de varier fortement en fonction des conditions climatiques, de critères caractérisant la maladie (contagiosité, morbidité, létalité), de ses modalités de transmission et de l'intensité et l'efficacité des moyens de lutte mis en œuvre.

A contrario, l'alimentation constitue un facteur endogène. Bien que l'aliment soit un input directement lié à la croissance des animaux, des désordres qualitatifs et/ou quantitatifs de la ration distribuée peuvent avoir des conséquences pathologiques. Par exemple, des carences d'apport en oligo-éléments de la ration augmentent la sensibilité des vaches laitières aux infections de la mamelle (Spears et Weiss, 2008). De même, les conditions de logement (type, ventilation, surface, entretien) représentent un facteur de risque de maladies (Assié et al., 2009a). Les caractéristiques propres de l'animal (race, âge, poids, sexe, stade de production) sont également liées au risque de maladies. Par exemple, leur relation avec l'incidence de troubles respiratoires a été mise en évidence chez le jeune bovin (Snowder et al., 2006). La récente cartographie du génome des animaux a permis de mettre en évidence des associations entre des gènes et des caractères de résistance aux maladies infectieuses, par exemple pour les mammites ou la tuberculose (Berry et al., 2011). Enfin, la conduite d'élevage, qui regroupe les objectifs fixés par l'éleveur (production, âge du premier vêlage, saison de vêlage, hors-sol) ainsi que ses pratiques d'élevage - incluant les mesures sanitaires médicales ou zootechniques préventives - sont de nature à agir 
sur l'incidence et la contagiosité des maladies Dans une étude récente menée en France, Raboisson et al. (2012) mettent ainsi en évidence une relation entre la dégradation de la qualité cytologique du lait (qui traduit la présence de mammites subcliniques) et l'âge au premier vêlage, le nombre de vaches, les taux de renouvellement et la présence d'un atelier viande au sein de l'exploitation laitière. En matière d'analyse des déterminants d'usage des antibiotiques, ces différents facteurs sont qualifiés d'endogènes, car ils relèvent directement des décisions de l'éleveur. La plupart des facteurs endogènes présentent la caractéristique d'être également des facteurs de production. Ceci implique que les maladies vont donc impacter la quantité et la qualité d'output, tout en étant liées à la quantité d'inputs employée, d'une part parce que ces inputs influent sur le risque de survenue de la maladie, d'autre part parce que la maladie influe sur la quantité d'input nécessaire pour la production.

\section{Impact des maladies sur le processus de production}

Les maladies infectieuses s'accompagnent de conséquences économiques défavorables sur la production des élevages :

- elles peuvent réduire la quantité ou la qualité de l'output (e.g. mortalité animale);

- elles peuvent diminuer la productivité des inputs utilisés dans le processus de production (e.g. baisse du Gain moyen quotidien [GMQ], ou hausse de l'Indice de consommation [IC]).

Il est possible de formaliser la production à l'échelle d'un atelier de production. L'approche développée par McInerney et al. (1992) intègre ainsi, par le biais de la fonction de production, les interactions entre les différents facteurs de production. Cette fonction postule une relation entre la quantité produite, notée $Q$ (lait ou viande par exemple) et la quantité d'inputs. Deux classes d'inputs peuvent être distinguées (Chi et al., 2002) :

- les facteurs variables directement liés à la production, notés $X$, qui disparaissent dans le processus de production (alimentation, autres charges variables, hormis les inputs de contrôle des dommages liés à la maladie, cf. ci-dessous) ;

- des facteurs de production qualifiés de quasi fixes, notés $K$ (logement, travail, cheptel...). Ces facteurs à l'origine des charges de structure, se dégradent à l'usage et doivent être entretenus et renouvelés; ils ne disparaissent pas dans le cycle de production de court à moyen terme.

La fonction de production en présence de maladie $M$ s'écrit alors :

$$
Q=f(X, M / K)
$$

L'hypothèse de productivité marginale décroissante de chaque input variable permet l'existence d'un maximum du profit à court terme. Cette hypothèse, en général vérifiée, signifie que l'augmentation de volume d'output se fait à taux décroissant avec l'augmentation des quantités employées de chaque input (figure 2). La formalisation retenue présente l'intérêt de décrire la technologie de production de manière très simplifiée, ce qui permet de comprendre comment les changements au sein du processus vont conditionner les choix de l'éleveur. L'apparition d'une maladie au sein du processus de production a pour conséquence une baisse de la productivité des facteurs de production. La fonction de production se déplace vers le bas avec l'intensité de la maladie $M$ (figure 2).

L'impact de la maladie $M$ sera variable en fonction des caractéristiques biologiques (e.g. faible ou forte mortalité), mais également en fonction de son expression dans le troupeau (e.g. faible ou forte contagiosité). Cela se traduit graphiquement par 


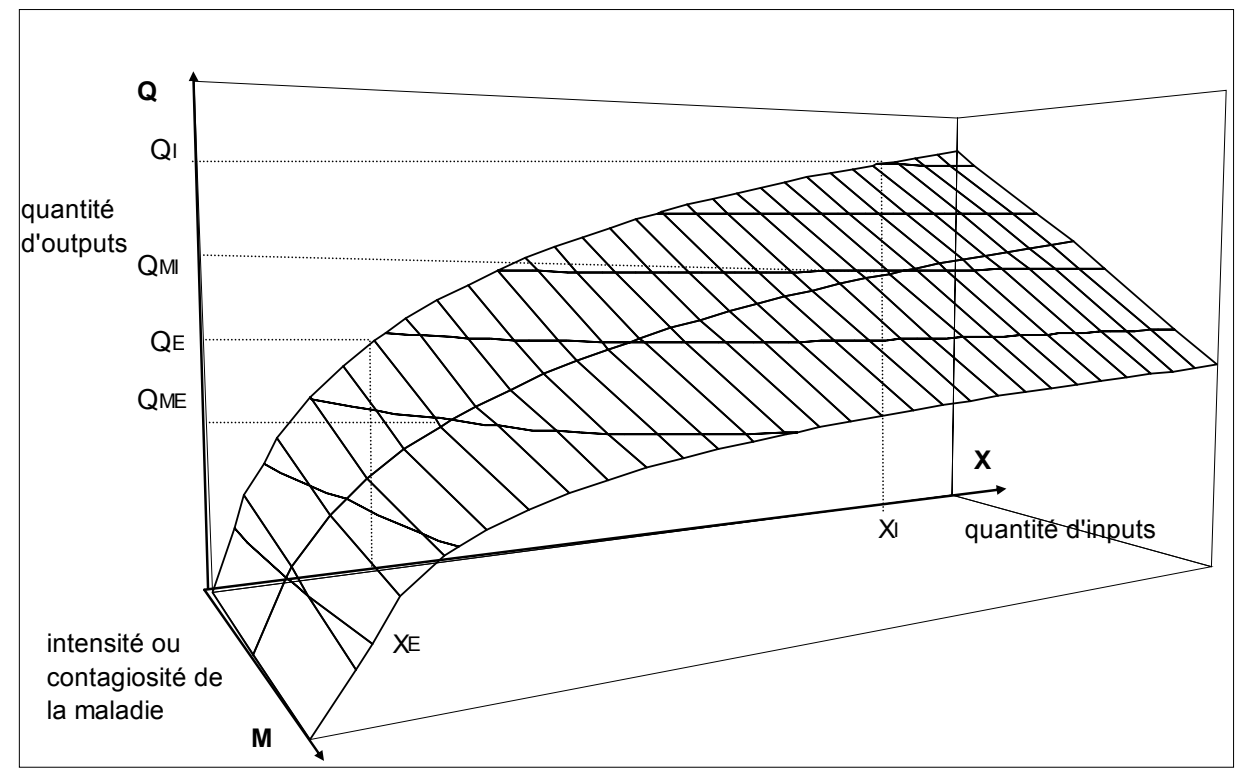

Note : Sur l'axe $x$ sont représentés les inputs $X\left(X\right.$, usage intensif, $X_{E}$ usage extensif) ; l'axe $z$ représente l'intensité ou la contagiosité de la maladie $M$; l'axe $y$ représente les outputs $Q$ (sans maladie $Q$ ou avec maladie $Q_{M}$ ).

Source : les auteurs.

un déplacement plus ou moins important de la courbe vers le bas, et une déformation de la courbe en présence de la maladie.

Par ailleurs, l'impact de la maladie sur un élevage utilisant de manière intensive l'input $\left(X_{I}\right)$ va entraîner des pertes supérieures à celles subies par un élevage faisant un usage plus faible de cet input $\left(X_{E}<X_{I}\right)$. L'intérêt de la maîtrise de la maladie varie ainsi en fonction des caractéristiques intrinsèques de la maladie, mais aussi en fonction de la conduite initiale du troupeau caractérisée par un usage plus ou moins intensif de l'input $X$. La réaction de l'éleveur à la survenue d'une maladie $M$, considérée ici comme une variable continue, peut comprendre à la fois un usage d'inputs de contrôle des dommages (médicaments et frais de prise en charge du sanitaire), notés $C D$, et un ajustement de la quantité d'inputs $X$.

\section{Rentabilité du traitement et sensibilité aux prix}

L'équation (1) traduit l'existence d'une relation entre les différents facteurs de production et la quantité d'outputs, mais ne renseigne pas directement sur le coût de la maladie. De plus, il est raisonnable de supposer qu'il est extrêmement difficile, sinon impossible, de ramener à zéro le niveau des pertes économiques induites par la maladie ; ceci implique que la variable pertinente n'est pas le coût total de la maladie mais le coût des pertes évitables avec un investissement en inputs de contrôle de dommages. Pour la plupart des maladies, l'évaluation des pertes induites par la maladie peut s'avérer complexe. La difficulté de cette estimation, associée au fait qu'elle ne permet pas de connaître le niveau optimal d'inputs de contrôle des dommages à utiliser, conduit à privilégier une méthode 
de calcul qui évalue le bénéfice de la maîtrise (Tisdell, 1995). Elle consiste à établir une relation entre le bénéfice (les pertes évitées) $(B)$ et le niveau des dépenses de maîtrise engagées $(C D)$, matérialisé sur la figure 3 :

$$
B=f(C D)
$$

Si la fonction de bénéfice croît à taux décroissant (du point $B$ au point $D$ ), le bénéfice maximal sera obtenu pour une valeur de $C D$ pour laquelle les bénéfices de la maîtrise seront équivalents aux coûts de maîtrise (point $C$ ). En pratique, il est possible que la maîtrise ne soit jamais rentable, en raison par exemple d'un coût fixe incompressible (e.g. une quantité minimale d'antibiotique à utiliser pour le traitement d'une maladie) supérieur au bénéfice maximal susceptible d'être retiré de la maîtrise. En élevage bovin laitier par exemple, l'utilisation des antibiotiques dans le traitement des mammites subcliniques des vaches en lactation n'est rentable que lorsque la fréquence des mammites est élevée (Swinkels et al., 2005). Dans ce cas, l'éleveur tolère les pertes, et la situation suboptimale ne requiert pas toujours le recours aux antibiotiques. Le bénéfice est maximisé quand $C D_{1}=Y_{1}$ est dépensé. La présence de coûts fixes engendre des seuils au-delà desquels l'utilisation d'antibiotique devient rentable. Un minimum $C D_{0}=Y_{0}$ de dépense doit être réalisé avant de pouvoir obtenir un bénéfice couvrant les dépenses engagées. Au-delà de $C D_{2}$, toute dépense de maîtrise supplémentaire n'est pas rentable. Dans ce cas, le seuil n'est pas associé à un coût initial incompressible. C'est la connaissance

Figure 3. Modèle coût-bénéfice de l'utilisation d'un traitement dans une situation où existe un effet de seuil

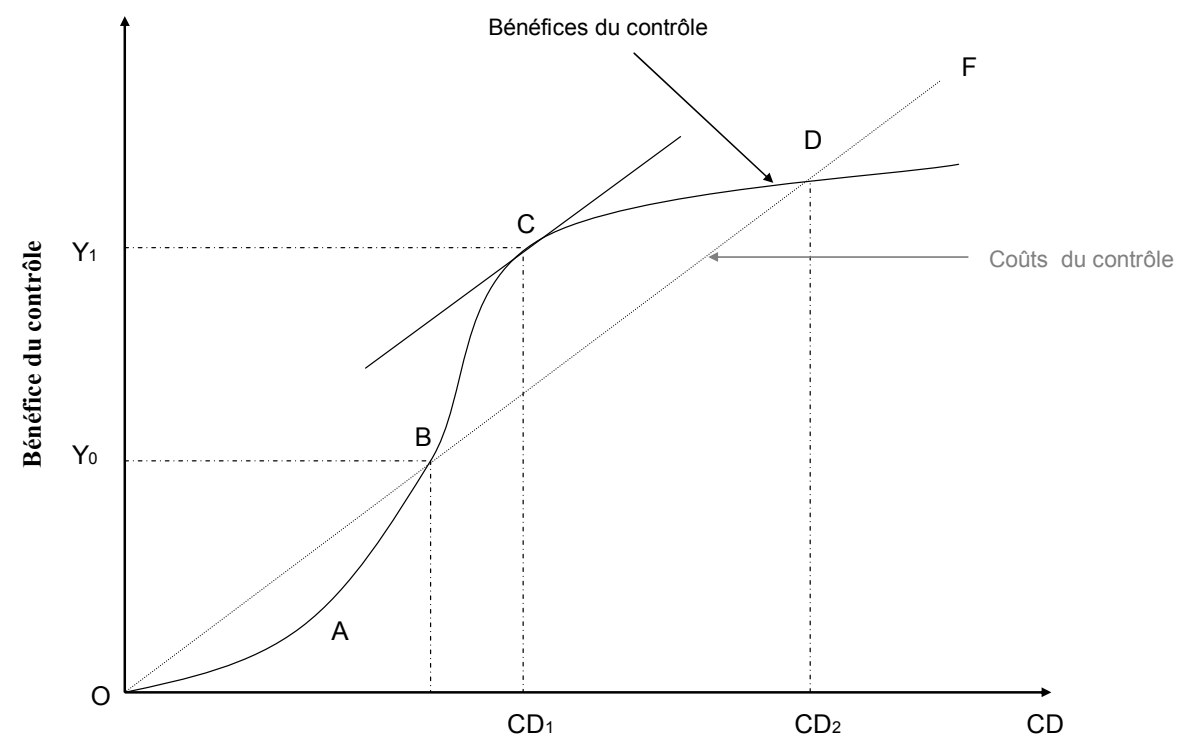

Niveau de dépense de contrôle des dommages

Note : Dans cette représentation, l'intensité de la maladie est constante. 
empirique de la maladie et de ses modalités de traitement qui permet de déterminer la forme de la fonction de bénéfice.

Dans la pratique, l'éleveur peut considérer les antibiotiques comme un facteur de production et décider de les utiliser en fonction du gain attendu du traitement. Le contrôle des dommages peut être considéré comme un processus économique, dans lequel l'augmentation des inputs vétérinaires génère un output supplémentaire qui prend la forme d'une réduction des pertes. La rationalité économique conduit à ne recourir aux antibiotiques que dans les situations où leur utilisation s'avère rentable, ce qui signifie qu'il existe un optimum économique de maîtrise des maladies, dépendant des prix de l'output et des inputs vétérinaires.

La nature économique de l'input " antibiotique », utilisé comme input de contrôle de dommages dans le cadre de la production, rend son niveau d'utilisation sensible au prix. Une diminution du prix des antibiotiques tend à accroître la quantité consommée. Une telle relation a par exemple été mise en évidence en production intensive, où l'introduction de génériques de fluoroquinolones sur le marché des antibiotiques autorisés en production aviaire s'est accompagnée d'un accroissement sensible des quantités consommées (Chevance et Moulin, 2009). En effet, l'introduction de génériques a pour effet de diminuer le prix des spécialités mises sur le marché, ce qui diminue le coût de contrôle des dommages. La courbe de la fonction de bénéfice se déplace vers la gauche, et entraîne une augmentation du bénéfice jusqu'à ce que le bénéfice marginal, décroissant avec le niveau de traitement, égale le coût marginal constant ou croissant. L'augmentation du prix de l'output, ou la diminution du prix des autres inputs, accroît également la consommation d'antibiotiques, en décalant la fonction de bénéfice du contrôle des dommages vers le haut. En considérant que la productivité croît à taux décroissant (figure 2), une augmentation du prix des produits est de nature à inciter l'éleveur à augmenter la quantité produite, impliquant une augmentation de la consommation d'inputs.

Du point de vue théorique, l'optimisation de l'usage des antibiotiques ne considère pas d'autres aspects sanitaires de la maladie (douleur, bien-être), et repose sur plusieurs hypothèses majeures. Pour décider, l'éleveur doit disposer de toutes les informations concernant la maladie (physiopathologie, épidémiologie) et les bénéfices qu'il peut retirer du traitement antibiotique. Ce cadre, bien que faiblement réaliste, demeure intéressant pour évaluer la capacité du traitement à diminuer les pertes imputables à la maladie, ainsi que pour étudier les variations des quantités utilisées dans un contexte donné (prix de marché, incidence de la maladie...). Il permet donc de bien comprendre la pertinence de mise en place de taxes, réglementations sur les quantités ou prix des antibiotiques. Cette approche occulte néanmoins les questions de substitution entre les facteurs de production, l'attitude de l'éleveur face au risque, l'influence de facteurs externes ou encore la présence d'externalités.

\section{Autres facteurs influençant la consommation d'antibiotiques}

En réalité, la demande d'antibiotiques par les éleveurs est influencée par plusieurs facteurs. Lors du choix de recourir (ou non) à un traitement antibiotique, toutes les alternatives ne sont pas connues et les conséquences évaluées par les éleveurs (Kristensen et Jakobsen, 2011). Dans le cas d'un traitement préventif, il est possible que la maladie ne se déclare pas, et les dépenses de maîtrise viennent alors diminuer le bénéfice de l'éleveur. Dans le cas d'un traitement curatif, une incertitude pèse également sur l'efficacité attendue du traitement. La décision de l'éleveur de recourir aux antibiotiques sera prise à 
la lumière de savoirs et savoir-faire, qui pourront être modulés par des facteurs intrinsèques au comportement de l'éleveur, ainsi que par l'environnement dans lequel il évolue. En élevage de ruminants, il a été montré que l'action en situation d'incertitude varie en fonction des différents leviers d'action dont dispose l'éleveur, qui seront mobilisés avec des logiques individuelles (Lemery et al., 2005). Des résultats récents, issus d'enquêtes réalisées en élevage de lapins et de volailles, mettent en évidence que le niveau de consommation d'antibiotiques demeure influencé par des facteurs autres que la survenue de la maladie, comme les caractéristiques de l'élevage et les pratiques de l'éleveur (Chauvin et al., 2012). Une enquête menée auprès de producteurs de dindes et de poulets a ainsi montré que $25 \%$ des élevages environ consommaient de un tiers à deux tiers de la quantité totale d'antibiotiques consommés par la population étudiée (106 bandes, échantillon représentatif des populations en France) (Chauvin et al., 2008). L'éleveur se construit donc une probabilité subjective d'apparition de maladie, qui varie en fonction de ses compétences techniques et de son attitude vis-à-vis du risque. Le rôle joué par l'aversion vis-à-vis du risque a été mis en évidence chez les éleveurs de bovins allaitants face à un aléa climatique ou sur le prix de la viande (Mosnier, 2009). Pour sécuriser son revenu, l'éleveur ayant une forte aversion vis-à-vis du risque préférera recourir aux antibiotiques. Cette aversion peut être à l'origine d'une surconsommation d'antibiotiques, notamment par des traitements préventifs. L'aversion vis-àvis du risque peut puiser son origine dans une sensibilité au stress ou dans des facteurs économiques : l'éleveur peut chercher à sécuriser son revenu en évitant une épidémie majeure au sein de son élevage, même si son revenu demeurera inférieur au revenu optimal (puisque la consommation d'antibiotiques sera en excès). Cette situation a par exemple été décrite dans une enquête réalisée en filière cunicole, montrant une corrélation négative entre le niveau de revenu et la consommation d'antibiotiques (Chauvin et al., 2011).

Ces arbitrages sont également influencés par l'environnement technicoéconomique de l'élevage, pour plusieurs raisons. Premièrement, la décision de l'éleveur de médicaliser ou non entraîne des conséquences à l'échelle collective. Par exemple, la sous-consommation médicamenteuse peut être responsable d'une augmentation de la prévalence des maladies contagieuses ; en effet, si le calcul privé d'un éleveur le conduit à ne pas maîtriser ces maladies, l'agent pathogène contagieux pourra se propager et affecter d'autres troupeaux. En ce sens, la sousconsommation collective peut être à l'origine d'une externalité négative (i.e. la dégradation de la santé et de la compétitivité du cheptel français, dégradation du bien-être des animaux). Tout d'abord, une série d'actions sanitaires pour la maîtrise des maladies contagieuses est coordonnée par les Groupements de défense sanitaire (GDS), qui peuvent inciter leurs adhérents à mettre en place des mesures faisant varier les consommations de médicaments (e.g. des programmes de vaccination). Puis, l'éleveur en tant que maillon de la chaîne de production de denrées alimentaires, peut voir ses choix être modérés par d'autres acteurs de la chaîne. Il peut adhérer à une filière, qui pourra tirer un avantage compétitif d'une utilisation encadrée. L'adhésion à la filière limitera les choix de l'éleveur. Par exemple, la production de produits transformés est souvent régie par un cahier des charges qui peut conduire l'éleveur à définir des stratégies sanitaires particulières (l'Agriculture Biologique et la consommation d'antibiotiques). En parallèle, il existe des éleveurs intégrés, dont la rémunération est principalement indexée sur leur compétence à produire des denrées 
à partir d'intrants (poussins, aliments, médicaments) fournis par l'intégrateur. Dans ce cas, il est tout à fait envisageable que la décision ou les modalités de traitement soient imposées par l'intégrateur. Enfin, les bénéfices économiques associés à la vente de médicaments pourraient expliquer des variations de consommation, malgré les régulations de ventes au sein du système pharmaceutique, décrit dans la figure 4.

Comme pour la demande, l'offre en antibiotiques subit l'influence de différents facteurs. Les antibiotiques sont distribués via le système pharmaceutique vétérinaire en suivant un flux marchand industries pharmaceutiques - propriétaires d'animaux, encadré depuis la Loi du 29 mai 1975 relative à la Pharmacie vétérinaire par des réglementations sur la production, la distribution et l'utilisation du médicament vétérinaire. La prescription ne peut être réalisée que par un vétérinaire. Elle se traduit par la rédaction d'une ordonnance, obligatoire avant la délivrance de tout médicament vétérinaire. La Loi n ${ }^{\circ}$ 75-409 du 29 mai 1975 relative à la Pharmacie vétérinaire et le Décret n 2007-596 du 24 avril 2007 précisent les conditions et modalités de prescription et délivrance au détail des médicaments vétérinaires. Jusqu'en 2007, la prescription ne pouvait s'exercer qu'au chevet de l'animal. Le Décret $n^{\circ} 2007$ 596 a légalisé les pratiques de prescription, hors examen clinique qui peut être actuellement réalisé après rédaction d'un bilan sanitaire annuel et d'un protocole de soins qui identifie les principales maladies affectant le troupeau, et leurs modalités de traitement. La distribution au détail (ou délivrance), qui pourrait être assimilée à l'offre sensu stricto, est octroyée à deux catégories d'ayants droit de plein exercice

Figure 4. Représentation schématique du système pharmaceutique vétérinaire

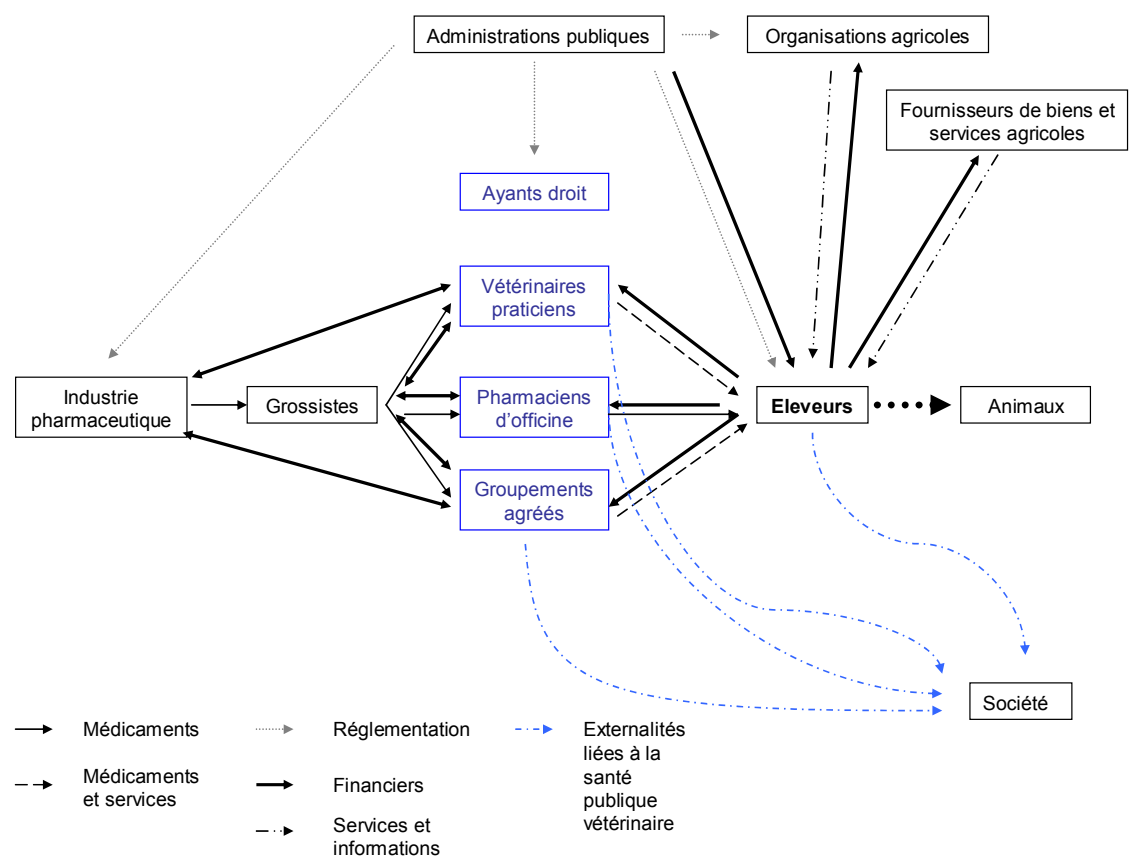

Source : les auteurs. 
(pharmaciens titulaires d'une officine et vétérinaires praticiens), ainsi qu'à un ayant droit d'exercice restreint (groupements de producteurs à vocation économique). Depuis 2007, suite à l'arrêt Riaucourt (Guillemot, 2009), les groupements de producteurs ne sont plus autorisés à acheter et délivrer les médicaments hors Plan sanitaire d'élevage (PSE) au nom du vétérinaire même s'ils emploient un vétérinaire. Cet arrêt a conduit à la création de sociétés par les vétérinaires salariés, en marge des groupements de producteurs, autorisées à délivrer les médicaments vétérinaires hors PSE. Les éleveurs adhérents aux groupements s'approvisionnent donc aujourd'hui à deux guichets : le groupement pour les médicaments à visée préventive, le vétérinaire libéral pour les médicaments à visée curative. En réalité, les pratiques de consommation de médicaments ont peu évolué ; la majorité des structures vétérinaires créées dépendent encore partiellement des groupements. À des fins de traçabilité, lors de la délivrance des médicaments soumis à ordonnance, le vétérinaire ou le pharmacien mentionne cette délivrance sur un registre sur lequel doivent être transcrites différentes informations relatives à l'identification du client, au médicament et au prescripteur. In fine, la position du vétérinaire, seul habilité à prescrire et autorisé à délivrer les antibiotiques, constitue une vraie singularité au sein du marché des antibiotiques. En théorie, la réglementation entourant la prescription des antibiotiques devrait garantir leurs bons usages, ceux-ci étant supervisés par le vétérinaire prescripteur, et limiter les consommations inutiles d'un point de vue médical. Mais la liberté de prescription, associée à la possibilité pour le vétérinaire de délivrer les antibiotiques, questionne les relations d'influence entre le praticien et l'éleveur.

Malgré des conditions d'accès limitées aux antibiotiques, le législateur a récemment implémenté des mesures ciblant les utilisateurs, mais aussi les prescripteurs et les dispensateurs pour réduire l'usage des antibiotiques. Si l'organisation du système pharmaceutique permet de contrôler qualitativement les flux d'antibiotiques, elle semble peiner à réguler la quantité consommée. En effet, la réglementation et ses évolutions de 1975 à 2007 devaient d'abord augmenter la traçabilité d'usage des médicaments vétérinaires, et garantir la sécurité des utilisateurs et des consommateurs. In fine, l'existence de ce système pourrait constituer un outil puissant, mobilisable pour réduire les consommations d'antibiotiques, mais ne semble pas pouvoir en l'état limiter les demandes des éleveurs. Pour le régulateur, l'enjeu consiste donc à mettre en place des mesures équilibrées concernant l'usage des antibiotiques. Cet équilibre s'avère nécessaire car paradoxalement la sous- ou sur-consommation d'antibiotiques peut influer sur la santé animale et sur la santé publique. Le risque sanitaire lié à l'antibiorésistance constitue une externalité négative qui justifie l'intervention des pouvoirs publics, qui mettent actuellement en place des leviers de maîtrise à l'aide d'approches réglementaire (LAA) et volontaire (Plan Ecoantibio). Le modèle conceptuel proposé dans la figure 1 précise les facteurs influençant l'usage des antibiotiques et les leviers de réduction associés. Les leviers institutionnels et économiques implémentés par les pouvoirs publics français sont l'objet d'étude de la section suivante.

\section{Pertinence des mesures récentes visant à réduire l'usage des antibiotiques en élevage}

L'existence de facteurs influençant la consommation des antibiotiques au niveau de l'offre et de la demande a conduit le régulateur à mettre en place des mesures spécifiques. Cette section discute la pertinence des mesures en respectant cette dichotomie. 


\section{Mesures ciblant la demande}

Les objectifs du Plan Ecoantibio et de l'article 49 de la LAA visent à réduire drastiquement l'usage des antibiotiques. À ce titre, le texte législatif stipule que «les bonnes pratiques d'élevage et les bonnes pratiques de prescription et d'utilisation de ces substances sont privilégiées ».

Six mesures de l'Axe 1 du Plan Ecoantibio («Promouvoir les bonnes pratiques et sensibiliser les acteurs aux risques liés à l'antibiorésistance et à la nécessité de préserver l'efficacité des antibiotiques ») ciblent spécifiquement l'augmentation de compétences des éleveurs. Une mesure de l'Axe 3 («Renforcer l'encadrement et réduire les pratiques à risque ») évoque la nécessité d'amélioration de la conduite d'élevage pour réduire les consommations d'antibiotiques.

La survenue de l'événement sanitaire ou son anticipation - est conditionnée par une conjonction de facteurs exogènes et endogènes défavorables. Bien que l'accès aux antibiotiques soit régulé, la mise en œuvre des traitements en l'élevage relève de l'initiative de l'éleveur. L'éleveur est en effet le décideur, mais également l'infirmier de ses animaux, et dispose d'une pharmacie qu'il peut utiliser, sous réserve de satisfaire à certaines exigences réglementaires. À ce titre, la mise en place de leviers visant à réduire la demande d'antibiotiques apparaît justifiée. La présente section discute de la portée de mesures visant à réduire la demande d'antibiotiques.

Réduire la consommation d'antibiotiques en augmentant les compétences individuelles des éleveurs

L'augmentation du degré de technicité des éleveurs constitue un levier indirect dans la réduction de consommation des antibiotiques, dans la mesure où elle peut permettre une réduction des usages non rationnels. Cette augmentation peut se faire selon deux modalités : un transfert technique de proximité d'une part, des actions coordonnées d'autre part. Ces deux modalités reposent sur le fait que l'élevage ne constitue pas une entité isolée, mais une structure en relation permanente avec des organismes divers - publics ou privés - qui jouent un rôle dans la prise en charge sanitaire (Chilonda, 2001 ; Raboisson et al., 2012). Dans le cas particulier des antibiotiques, l'encadrement technique fourni par le vétérinaire prescripteur va influer sur leur usage (Kaneene et Miller, 1992). D'autres acteurs (techniciens, inséminateurs, nutritionnistes...), entretenant des relations privilégiées avec l'éleveur, peuvent également intervenir dans le processus de décision, voire même prendre directement cette décision. Indépendamment des vecteurs d'informations, l'éducation des éleveurs en matière de bon usage des antibiotiques pourrait permettre de rationaliser les traitements.

Dans certains cas, les institutions peuvent être les moteurs d'actions collectives, dont l'objectif est de coordonner les actions individuelles des producteurs afin de répondre à une problématique collective (Ostrom, 1990). En médecine humaine par exemple, des actions collectives de sensibilisation des consommateurs, via des campagnes de communication coordonnées par les pouvoirs publics (slogans du type «Les antibiotiques, c'est pas automatique ») ont permis une diminution des volumes d'antibiotiques consommés (Sabuncu et al., 2009). L'éducation des praticiens en petits groupes, animés par un expert, débouche également sur une diminution significative des consommations de médicaments (Madridejos-Mora et al., 2004 ; Ranji et al., 2008). A contrario, la rédaction de guides de bonnes pratiques largement diffusés, dont l'objectif est de rappeler les bons et mauvais usages des antibiotiques, semble produire des résultats moins tangibles (Al-Momany et al., 2009 ; Faryna et al., 1987). 
L'impact d'actions collectives concernant la consommation d'antibiotiques demeure mal connu en productions animales. Historiquement, la lutte contre les maladies infectieuses reste basée sur l'adhésion des éleveurs aux programmes de prophylaxie coordonnés par les GDS. L'adhésion des éleveurs aux GDS, avant tout mue par des intérêts individuels (diminution de l'incidence de maladie), a néanmoins deux répercussions collectives concernant la réduction d'usage d'antibiotiques. Elle permet d'une part de modifier le contexte épidémiologique et in fine la consommation d'antibiotiques (Rat-Aspert et Fourichon, 2010). Elle permet également de délivrer un message standardisé à tous les adhérents sur le bon usage des antibiotiques, par exemple à travers des programmes de formations du type « éleveur-infirmier ». La contribution d'actions volontaires coordonnées à la diminution de consommation d'antibiotiques en élevage, théoriquement bénéfique, reste encore à documenter.

\section{Réduire la consommation d'antibiotiques en substituant d'autres facteurs de production}

Il existe, au sein d'un système d'élevage donné, un niveau basal de maladies, qui peut être qualifié de «norme sanitaire » (Raboisson et al., 2012), et qui conduit à définir un niveau normé de consommation d'antibiotiques. Cette norme sanitaire est associée à des facteurs (déjà discutés dans la section 1), sur lesquels l'éleveur ne peut pas influer.

Les déterminants de la consommation qui nous préoccupent ici sont les facteurs endogènes, dans la mesure où une meilleure maîtrise de ces derniers par l'éleveur est de nature à faire varier le niveau de consommation d'antibiotiques dans l'exploitation. La majorité des facteurs de risque recensés constitue des facteurs de production. Se pose alors la question de la substituabilité entre ces différents facteurs endogènes. Est-il par exemple possible d'atteindre un même niveau de production en utilisant des niveaux différents d'inputs ? Un exemple courant de substitution est le recours à la prophylaxie médicale (vaccination, antibioprévention) pour diminuer un risque de maladie lors de mauvaises conditions d'élevage.

La relation de substitution entre facteurs de production s'envisage facilement entre ressources sanitaires préventives et facteurs de production non sanitaires (alimentation, logement), sans a priori sur la nature parfaite ou non parfaite de la relation (plus d'unités d'un facteur employées pour compenser le manque d'un autre). Elle s'envisage aussi aisément entre différents types de ressources sanitaires préventives (vaccin A ou vaccin B ou antibioprévention). Des substitutions entre inputs médicamenteux préventifs et inputs curatifs sont également possibles, comme l'illustre l'intérêt de la vaccination pour diminuer le nombre de traitements curatifs des maladies respiratoires des jeunes bovins (Assié et al., 2009b). La maîtrise des facteurs de risque endogènes impacte donc qualitativement (sévérité) et quantitativement (incidence, contagiosité) la maladie, et in fine les consommations d'antibiotiques. Cette relation a également été mise en évidence en production porcine, dans une enquête rapportant de plus faibles consommations antibiotiques dans les élevages mettant en place des mesures de biosécurité (Laanen et al., 2013). La substitution entre inputs médicamenteux et autres inputs est possible mais nécessite une anticipation de l'événement sanitaire. En cas d'apparition de troubles sanitaires, la demande de traitement curatif devient rigide. Dans ce cas, le niveau de consommation des autres facteurs ne varie que faiblement. L'anticipation repose en partie sur les compétences individuelles des éleveurs et leur capacité (financières, logistiques...) à mettre en place des substitutions. 
Réduire la consommation d'antibiotiques en faisant varier les prix

Lorsqu'une substitution est possible, la question de son intérêt économique demeure. Les difficultés d'estimation de l'intérêt économique de mesures zootechniques, la volatilité des prix des produits et des facteurs de production, constituent autant d'incertitudes qui viennent limiter les alternatives non médicales aux antibiotiques à court terme, même si elles semblent socialement pertinentes compte tenu des externalités liées à l'usage des antibiotiques en élevage. La substituabilité entre facteurs de production favorise le recours à des inputs à retour sur investissement rapide tels que les antibiotiques, dont l'efficacité est garantie dans des conditions d'utilisation standard et limite l'incertitude sur le revenu agricole. En outre, les antibiotiques assurent un contrôle de dommage à court terme, tandis que beaucoup d'autres facteurs l'assurent à long et moyen termes.

En raison des contraintes budgétaires propres à toute exploitation agricole, les ressources monétaires sont allouées aux stratégies de maîtrise de la maladie caractérisées par la rentabilité la plus élevée. Cette contrainte budgétaire peut limiter l'éleveur dans le choix de certains investissements coûteux (mais pouvant néanmoins s'avérer rentables à long terme, comme la réfection d'un bâtiment) et rigidifier encore un peu plus la demande en antibiotique. Enfin, la contrainte budgétaire conduit l'éleveur à favoriser des investissements caractérisés par une probabilité élevée de retour sur investissement. Si les antibiotiques n'assurent aucun retour sur investissement en l'absence de maladies à venir, leur efficacité autorise un niveau de maîtrise de la maladie très satisfaisant en cas de survenue, et le recours aux antibiotiques est aisé techniquement. In fine, les antibiotiques s'avèrent de puissants outils de contrôle de dommages, fortement résistants à la contrainte budgétaire.

\section{Mesures visant l'offre des antibiotiques}

La décision du législateur de mettre en place des mesures incitatives, mais également coercitives ciblant directement les prescripteurs et les ayants droit, matérialise la volonté des pouvoirs publics de maîtriser de possibles dérives au sein même du système de distribution du médicament vétérinaire. Quatorze mesures du Plan Ecoantibio ciblent les vétérinaires, dont une majorité est également inscrite dans la LAA. Les mesures réglementaires majeures sont étudiées dans cette soussection.

\section{Réduire les usages en encadrant la prescription des antibiotiques}

L'objectif global de réduction de $25 \%$ de l'usage de l'ensemble des antibiotiques, fixé par le Plan Ecoantibio, est réaffirmé par la LAA, qui prévoit une réduction de $25 \%$ entre 2013 et 2016 de l'usage des fluoroquinolones et céphalosporines de $3^{\mathrm{e}}$ et $4^{\text {e }}$ générations. La fixation d'un objectif global, identique pour toutes les espèces animales concernées, laisse perplexe quant à son applicabilité. Certaines filières (principalement d'élevage intensif), fortement consommatrices d'antibiotiques, peuvent contribuer fortement à la réalisation de cet objectif, alors même que des filières de production plus extensives ne disposeront que de peu de leviers de réduction de leurs consommations d'antibiotiques. Enfin, l'impact sur l'antibiorésistance de telles réductions d'usage demeure encore à évaluer.

Un décret en cours d'élaboration, correspondant à la mesure 26 du Plan Ecoantibio, prévoit en outre un encadrement strict de la prescription des antibiotiques d'importance critique. S'il est aisément possible d'imaginer que ces mesures contraignantes vont limiter la consommation des familles d'antibiotiques ciblées, leur impact concernant les mésusages ou les surconsommations demeure incertain. En effet, pour une 
affection donnée, il est probable qu'une famille d'antibiotique soit substituée à une autre, ce qui au final (i) n'entraînera pas de baisses réelles d'usage, et (ii) n'aura que peu d'effet sur l'externalité (antibiorésistance), du fait de l'existence de résistances croisées entre les différentes familles d'antibiotiques (Carattoli, 2009).

\section{Réduire les usages en encadrant les pratiques commerciales associées aux antibiotiques}

L'aménagement de mesures visant à restreindre les incitations à la prescription ou à la délivrance à tous les étages du système pharmaceutique, recommandé en France par le Conseil général de l'alimentation, de l'agriculture et des espaces (CGAAER) (Dahan, 2013), apparaît aujourd'hui dans l'article 48 de la LAA. Les mesures préconisées visent à mieux réguler les relations financières, directes ou indirectes, entre les fabricants de médicaments et les ayants droit.

La loi prévoit d'abord l'interdiction du versement d'avantages en nature (matériels, voyages...) aux ayants droit, mais également aux sociétés les représentant ainsi qu'aux étudiants vétérinaires. Elle prévoit également des mesures visant à interdire toute possibilité d'incitation à l'achat d'antibiotiques par le biais de remises « croisées ${ }^{3}$ sur d'autres catégories de médicaments. La réglementation vise également à interdire la conclusion de contrats de coopération commerciale concernant la vente d'antibiotiques. Enfin, la Loi prévoit l'interdiction de toute forme de remises, rabais ou ristournes ${ }^{4}$ octroyées lors de l'achat d'antibiotiques

3. L'ayant droit toucherait alors la remise initialement reliée à l'achat d' antibiotiques en cas d'achat d'un autre médicament non antibiotique.

4. Les remises (ou remises arrière ou marges arrière) sont des rémunérations versées sous différentes formes par les fournisseurs aux ayants droit avec lesquels ils concluent des accords commerciaux. (toutes familles d'antibiotiques confondues). L'interdiction de remises sur toutes les familles d'antibiotiques présente d'un point de vue théorique l'intérêt de limiter les incitations à la prescription, mais également de décourager les substitutions entre familles d'antibiotiques (à la différence de mesures spécifiques à certaines molécules). Toutefois, le dispositif législatif présuppose que les remises associées à la vente d'antibiotiques influent sur le prix de vente facturé à l'éleveur, ce qui reste dans les faits à établir. Dans l'hypothèse où les remises ne sont pas - ou peu - répercutées sur le prix final, il est peu probable que cette mesure influence réellement le prix réel de vente des antibiotiques appliqué aux éleveurs. Le degré de réintégration des remises dans le prix de vente au consommateur varie fortement en fonction des filières de production considérées. Par ailleurs, l'encadrement des marges des intermédiaires présuppose que le vétérinaire soit en mesure de persuader l'éleveur d'utiliser des antibiotiques au-delà de son optimum de profit. Cette hypothèse reste également à vérifier.

La LAA prévoit enfin l'interdiction de conditions particulières de ventes, c'està-dire que le vendeur (l'industrie pharmaceutique et les ayants droit) devra afficher un prix unique, valable pour l'ensemble de ses clients. Cette interdiction semble théoriquement opportune, car elle permettrait de pénaliser les gros consommateurs d'antibiotiques, qui bénéficieraient de tarifs préférentiels. Néanmoins, cette mesure ne règle pas totalement la question de la concurrence entre ayants droit, cette dernière pouvant conduire à terme à une chute du prix des antibiotiques (la marge reste librement fixée par l'ayant droit), qui s'accompagnerait une augmentation des volumes commercialisés.

Finalement, l'ensemble de ces mesures législatives ne règle donc pas totalement la question fondamentale de la fixation 
du prix de vente. Il n'existe pas à ce jour de données permettant d'évaluer la pertinence des mesures évoquées ci-dessus. La mise en place de taxes et l'administration du prix de vente des antibiotiques constituent deux alternatives étonnamment peu étudiées. Un système de taxe pigovienne (Pigou, 1920) pourrait permettre d'internaliser l'externalité (antibiorésistance). Une telle taxe est théoriquement égale au dommage des effets externes provoqués par l'utilisation d'une unité supplémentaire d'antibiotique. Il est possible que la taxe pigovienne n'ait qu'un effet limité sur la réduction de la consommation d'antibiotiques, car leur demande en productions animales est probablement rigide ; en revanche, elle permettrait de maximiser l'utilité de la consommation. Sa mise en place nécessite néanmoins une évaluation préalable des dommages, ce qui s'avère complexe dans le cas de l'antibiorésistance. Une alternative consisterait à fixer une taxe sur les antibiotiques, dont le montant pourrait être fixé en fonction des coûts de développements supportés par les industries pharmaceutiques (Vagsholm et Hojgard, 2010). La taxe, supportée soit par les industriels, soit par les consommateurs, pourrait être redistribuée pour encourager la recherche en antibiologie. Une administration du prix des antibiotiques, destinée à maintenir les prix pratiqués à un niveau élevé, adresserait au marché un signal équivalent.

\section{Réduire les usages en réduisant \\ la dépendance économique}

des vétérinaires aux ventes d'antibiotiques

La délivrance au détail se répartit de manière relativement inégale entre les ayants droit, l'essentiel des volumes d'antibiotiques (environ $80 \%$ ) étant délivré par les vétérinaires. La relation marchande entre le prescripteur-délivreur (le vétérinaire) et ses clients (les éleveurs) soulève la question du conflit d'intérêts. Le vétérinaire pourrait en effet être incité à accroître ses prescriptions de médicaments, de manière à augmenter le profit retiré de son activité commerciale d'ayant droit. La simultanéité d'exercice de la prescription et de la délivrance des antibiotiques par le vétérinaire pourrait ainsi engendrer une situation propice à la surconsommation d'antibiotiques. Le découplage prescription-délivrance des antibiotiques a été repoussé en France, bien qu'il soit en vigueur dans d'autres pays de l'Union européenne (Rosbach, 2012). Ce découplage semble produire des résultats controversés (Dahan, 2013). Il permet bien de solutionner la problématique du conflit d'intérêts, mais la dépendance financière des prescripteurs vis-à-vis des éleveurs pourrait conduire, dans un marché concurrentiel de l'offre médicale, les vétérinaires à la prescription de « complaisance». La menace de découplage qui a pesé durant le travail législatif pourrait avoir joué positivement sur la réduction de consommation d'antibiotiques. Une évaluation économique de l'intérêt de cette mesure pourrait s'avérer intéressante à la lumière de la non-atteinte des objectifs de réduction. L'investissement des vétérinaires dans des stratégies visant à diversifier leurs sources de revenus, telles que la vente de services, pourrait être un témoin intéressant de leur dépendance financière à la vente d'antibiotiques, et éclairer la réalité du conflit d'intérêts.

Au-delà de la dualité d'exercice du vétérinaire, à la fois prescripteur et dispensateur d'antibiotiques, la relation client-fournisseur qui lie l'éleveur et le vétérinaire mériterait d'être questionnée au regard de la consommation d'antibiotiques. En médecine humaine, la relation marchande liant le prescripteur et le patient, et la volonté de ne pas s'écarter de la pratique dominante constituent deux facteurs explicatifs de recours non justifié aux antibiotiques (Rosman, 2010). La préférence de certains praticiens pour des médicaments avec « effet de démonstration », afin que 
les patients aient l'impression d'avoir « réellement reçu des soins » a également été observée (Orzech et Nichter, 2008). La pression exercée par les patients sur le médecin pour orienter la prescription d'antibiotiques a également été décrite (Avorn et Solomon, 2000 ; Orzech et Nichter, 2008). Il est vraisemblable que de tels phénomènes existent en médecine vétérinaire. En agriculture s'ajoute le risque économique associé à la non-utilisation d'antibiotiques, qui peut conduire à une surmédicalisation des animaux : l'utilisation d'antibiotiques pourrait jouer un effet d'assurance, en diminuant le risque d'échec thérapeutique, lié à des difficultés de diagnostic. Les pressions possiblement exercées par les éleveurs pour avoir accès aux antibiotiques n'ont à notre connaissance pas fait l'objet d'études à ce jour, et aucun dispositif réglementaire actuel ne semble les considérer.

$*$
$* \quad *$

L'activation de leviers (incitations, réglementation) permettant de substituer des mesures préventives aux antibiotiques peut présenter un intérêt, dans la mesure où le coût des alternatives est inférieur à celui des antibiotiques, car elle permet d'améliorer la compétitivité des élevages et de réduire la consommation d'antibiotiques. Deux types d'approches, complémentaires, ressortent de l'analyse proposée. Les approches réglementaires ont pour objectif de contraindre l'utilisation des antibiotiques, et de limiter l'influence des pratiques commerciales sur leur consommation. Ce type de mesures ne considère pas la question de la nécessité d'usage comme centrale, mais poursuit l'objectif de limiter l'usage et les incitations à l'usage, toutes choses égales par ailleurs. Si l'efficacité de telles mesures à court terme peut s'avérer importante, il convient néanmoins d'évaluer les possibilités de contournement. Les approches volontaires regroupent à la fois des initiatives privées et payantes (l'éleveur finance l'augmentation de son niveau de compétences sanitaires) et des initiatives collectives (adhésion à un groupement ou engagement dans un cahier des charges). Même si leurs effets restent plus difficiles à mesurer, ces approches présentent l'avantage de sensibiliser les parties prenantes à la problématique (particulièrement les éleveurs) qui peuvent internaliser l'externalité dans leur processus de prise de décision. Dans le cas de l'utilisation des antibiotiques, toutes les initiatives permettant de réduire l'incidence des maladies sont vertueuses, car elles permettent d'augmenter la compétitivité des élevages, en diminuant les consommations antibiotiques.

La réduction de l'usage des antibiotiques, à travers les différents outils présentés dans cet article, poursuit un objectif de lutte contre l'antibiorésistance. Parmi tous les dispositifs de lutte contre l'antibiorésistance mis en œuvre ou envisagés par les autorités sanitaires, on peut s'étonner de l'absence de réflexions sur l'administration des prix et de dispositifs s'inspirant de la taxe pigovienne. Si l'antibiorésistance constitue une réalité, la mesure des dommages qu'elle entraîne doit être menée prioritairement. Comme ces dommages sont décalés dans le temps, un consensus d'experts et de citoyens doit être recherché concernant les hypothèses de calcul, en particulier concernant le taux d'actualisation des dommages futurs. Simultanément, les déterminants de l'antibiorésistance se doivent d'être mieux investigués, en évaluant l'ensemble des stratégies de traitements possibles (e.g. curatif $v$ s préventif ; faible ou forte posologie ; choix d'un antibiotique critique ou non). Ces diverses connaissances demeurent nécessaires pour éclairer la prise de décision publique. Le Plan Ecoantibio fournit un objectif indicatif de réduction de $25 \%$ des consommations d'antibiotiques. Il est principalement 
un signal destiné à mettre en avant l'importance de cette question, mais sa portée sur les choix individuels demeure limitée. Les mesures de persuasion et de formation sont nécessaires à une réduction des consommations d'antibiotiques. Elles ne sont pas toutefois suffisantes tant que la dernière unité consommée apparaît rentable au décideur. Inversement, une internalisation de l'externalité par une taxe constitue également une incitation pour les usagers à acquérir les compétences pour s'adapter à cette taxe en baissant sa consommation au moindre coût.

\section{RÉFÉRENCES BIBLIOGRAPHIQUES}

Al-Momany N. H., Al-Bakri A. G., Makahleh Z. M., Wazaify M. M. (2009). Adherence to international antimicrobial prophylaxis guidelines in cardiac surgery: a Jordanian study demonstrates need for quality improvement. Journal of Managed Care Pharmacy, $\mathrm{n}^{\circ} 15$, pp. 262-271.

Angulo F. J., Baker N. L., Olsen S. J., Anderson A., Barrett T. J. (2004). Antimicrobial use in agriculture: controlling the transfer of antimicrobial resistance to humans. Seminar in Pediatric Infectious Diseases $\mathrm{n}^{\circ} 15$, pp. 78-85.

Assie S., Bareille N., Beaudeau F., Seegers H. (2009a). Management- and housing-related risk factors of respiratory disorders in nonweaned French Charolais calves. Preventive Veterinary Medicine, ${ }^{\circ}$ 91, pp. 218-225.

Assie S., Seegers H., Makoschey B., DesireBousquie L., Bareille N. (2009b). Exposure to pathogens and incidence of respiratory disease in young bulls on their arrival at fattening operations in France. Veterinary Record, $\mathrm{n}^{\circ} 165$, pp. 195-199.

Avorn J., Solomon D. H. (2000). Cultural and economic factors that (mis)shape antibiotic use: the nonpharmacologic basis of therapeutics. Annals of Internal Medicine, $\mathrm{n}^{\circ} 133$, pp. 128-135.

Bentley R., Bennett J. W. (2003). What is an antibiotic ? Revisited. Advances in Applied Microbiology, $\mathrm{n}^{\circ}$ 52, pp. 303-331.

Berry D. P., Bermingham M. L., Good M., More S. J. (2011). Genetics of animal health and disease in cattle. Irish Veterinary Journal, $\mathrm{n}^{\circ} 64$, p. 5 .
Carattoli A. (2009). Resistance plasmid families in Enterobacteriaceae. Antimicrobial Agents Chemotherapy, $\mathrm{n}^{\circ}$ 53, pp. 2227-2238.

Cernicchiaro N., Renter D. G., White B. J., Babcock A. H., Fox J. T. (2012). Associations between weather conditions during the first 45 days after feedlot arrival and daily respiratory disease risks in autumn-placed feeder cattle in the United States. Journal of Animal Science, $\mathrm{n}^{\circ}$ 90, pp. 1328-1337.

Chauvin C., Croisier A., Tazani F., Balaine L., Eono F., Salaun-Huneau A., Le Bouquin S., (2011). Utilisation des antibiotiques en filière cunicole : enquête en élevages 20092010. Journées de la Recherche Cunicole, 22-23 novembre 2011, Le Mans.

Chauvin C., Le Bouquin S., Sanders P. (2012). Usage des antibiotiques en filières porcine, avicole et cunicole en France. Résultats d'enquêtes. Bulletin épidémiologique santé animale-alimentation, pp. 12-15.

Chauvin C., Querrec M., Perot A., Guillemot D., Sanders P. (2008). Impact of antimicrobial drug usage measures on the identification of heavy users, patterns of usage of the different antimicrobial classes and time-trends evolution. Journal of Veterinary Pharmacology and Therapeutics, $\mathrm{n}^{\circ}$ 31, pp. 301-311.

Chevance A., Moulin G. (2009). Suivi des ventes de médicaments vétérinaires contenant des antibiotiques en France en 2008. Fougères, AFSSA, rapport annuel, 44 p.

Chevance A., Moulin G. (2012). Suivi des ventes de médicaments vétérinaires contenant des antibiotiques en France en 2011. Paris, Anses, rapport annuel, $72 \mathrm{p}$. 
RECHERCHE

Guillaume LHERMIE, Didier RABOISSON, Stéphane KREBS, Pierre DUPRAZ

Chi J., Weersink A., VanLeeuwen J. A., Keefe G. P. (2002). The Economics of Controlling Infectious Diseases on Dairy Farms. Canadian Journal of Agricultural Economics/Revue canadienne d'agroéconomie, $\mathrm{n}^{\circ} 50$, pp. 237-256.

Chilonda P., Van Huylenbroeck G. (2001). A conceptual framework for the economic analysis of factors influencing decisionmaking of small-scale farmers in animal health management. Revue Scientifique et Technique de l'Office International des Épizooties, $\mathrm{n}^{\circ} 20$, pp. 687-700.

Dahan M. (2013). Encadrement des pratiques commerciales pouvant influencer la prescription des antibiotiques vétérinaires. Rapport du Conseil général de l'agriculture, de l'alimentation et des espaces ruraux, $123 \mathrm{p}$.

European Union (2003). Regulation (EC) No. 1831/2003 of the European Parliament and of the Council of 22 September 2003 on additives for use in animal nutrition. Official Journal of the European Union, 15 p.

Faryna A., Wergowske G. L., Goldenberg K. (1987). Impact of therapeutic guidelines on antibiotic use by residents in primary care clinics. Journal of General Internal Medicine, $\mathrm{n}^{\circ} 2$, pp. 102-107.

Guillemot P., Vandaële E. (2009). L'arrêt Riaucourt et ses effets. Bulletin de l'Académie Vétérinaire de France, ${ }^{\circ} 162$, pp. 171-180.

Kaneene J. B., Miller R. (1992). Description and evaluation of the influence of veterinary presence on the use of antibiotics and sulfonamides in dairy herds. Journal of American Veterinary Medicine Association, $\mathrm{n}^{\circ} 201$, pp. 68-76.

Kristensen E., Jakobsen E. B. (2011). Challenging the myth of the irrational dairy farmer ; understanding decision-making related to herd health. New Zealand Veterinary Journal, n5 59 , pp. 1-7.

Laanen M., Persoons D., Ribbens S., de Jong E., Callen B., Strubbe M., Maes D., Dewulf J. (2013). Relationship between biosecurity and pro-duction/antimicrobial treatment characteristics in pig herds. Veterinary Journal., $\mathrm{n}^{\circ} 198$, pp. 508-512.

Lemery B., Ingrand S., Dedieu B., Dégrange B. (2005). Agir en situation d'incertitude : le cas des éleveurs de bovins allaitants. Économie rurale, $\mathrm{n}^{\circ} 288$, pp. 57-69.

Lhermie G. (2010). Demande de médicaments en productions animales. Montpellier, Faculté de sciences économiques, mémoire de fin d'études, 90 p.

Madridejos-Mora R., Amado-Guirado E., Perez-Rodriguez M. T. (2004). Effectiveness of the combination of feedback and educational recommendations for improving drug prescription in general practice. Medical Care, $\mathrm{n}^{\circ} 42$, pp. 643-648.

McInerney J. (1996). Old ecocomics for new problems - livestock disease: presidential address. Journal of Agricultural Economics, $\mathrm{n}^{\circ} 47$, pp. 295-314.

McInerney J. P., Howe K. S., Schepers J. A. (1992). A framework for the economic analysis of disease in farm livestock. Preventive Veterinary Medicine, $\mathrm{n}^{\circ}$ 13, pp. 137-154.

Ministère de l'Agriculture de l'Alimentation de la Pêche et de la Ruralité (2011). Plan National De Réduction des Risques d'Antibiorésistance en Médecine Vétérinaire ECOANTIBIO 2017. Document de synthèse, 32 p.

Mosnier C. (2009). Adaptation of suckler cow farms to weather and beef price risks: modelling approaches. Paris, Agroparis-Tech, thèse de doctorat d'économie, $182 \mathrm{p}$.

Moulin G., Cavalie P., Pellanne I., Chevance A., Laval A., Millemann Y., Colin P., Chauvin C. (2008). A comparison of antimicrobial usage in human and veterinary medicine in France from 1999 to 2005. Journal Antimicrobial Chemotherapy, $\mathrm{n}^{\circ}$ 62, pp. 617-625.

Orzech K. M., Nichter M. (2008). From Resilience to Resistance: Political Ecological Lessons from Antibiotic and Pesticide Resistance. Annual Review of Anthropology, $\mathrm{n}^{\circ} 37$, pp. 267-282.

Ostrom E. (1990). Governing the Commons: The Evolution of Institutions for Collective Action. Cambridge University Press, 60 p.

Pigou A. (1920). The economics of welfare (1932). London, McMillan\&Co.

Raboisson D., Derville M., Herman N., Cahuzac E., Sans P., Allaire G. (2012). Herd-level and territorial-level factors influencing average herd somatic cell count in 
France in 2005 and 2006. Journal of Dairy Researc, ${ }^{\circ} 79$, pp. 324-332.

Ranji S. R., Steinman M. A., Shojania K. G., Gonzales R. (2008). Interventions to reduce unnecessary antibiotic prescribing: a systematic review and quantitative analysis. Medical Care $\mathrm{n}^{\circ} 46$, pp. 847-862.

Rat-Aspert O., Fourichon C. (2010). Modelling collective effectiveness of voluntary vaccination with and without incentives. Preventive Veterinary Medicine, $\mathrm{n}^{\circ}$ 93, pp. 265-275.

Rosbach A. (2012). On the Microbial ChallengeRising threats from Antimicrobial Resistance European Parliament. Report, 24 p.

Rosman S. (2010). Les pratiques de prescription des antibiotiques en médecine générale en France et aux Pays-Bas. Revue Sociologie Santé $n^{\circ}$ spécial Médicaments et Société, $\mathrm{n}^{\circ} 30$, pp. 81-99.

Sabuncu E., David J., Bernede-Bauduin C., Pepin S., Leroy M., Boelle P. Y., Watier L., Guillemot D. (2009). Significant reduction of antibiotic use in the community after a nationwide campaign in France, 2002-2007. PLOS Medicine, ${ }^{\circ}$ 6, pp. 2-9.

Singer R. S., Finch R., Wegener H. C., Bywater R., Walters J., Lipsitch M. (2003). Antibiotic resistance--the interplay between antibiotic use in animals and human beings. Lancet Infect Dis, $\mathrm{n}^{\circ}$ 3, pp. 47-51.
Snowder G. D., Van Vleck L. D., Cundiff L. V., Bennett G. L. (2006). Bovine respiratory disease in feedlot cattle: environmental, genetic, and economic factors. Journal of Animal Science, $\mathrm{n}^{\circ}$ 84, pp. 1999-2008.

Spears J. W., Weiss W. P. (2008). Role of antioxidants and trace elements in health and immunity of transition dairy cows. Veterinary Journal, $\mathrm{n}^{\circ}$ 176, pp. 70-76.

Swinkels J. M., Hogeveen H., Zadoks R. N. (2005). A partial budget model to estimate economic benefits of lactational treatment of subclinical Staphylococcus aureus mastitis. $J$ Dairy Science, ${ }^{\circ}{ }^{8} 8$, pp. 4273-4287.

Tisdell C. (1995). Assessing the Approach to Cost-Benefit Analysis of Controlling Livestock Diseases of McInerney and Others. Ed. University of Queensland, Research Papers and Reports, Animal Health Economics, $\mathrm{n}^{\circ} 3$, Economics, $22 \mathrm{p}$.

Vagsholm I., Hojgard S. (2010). Antimicrobial sensitivity-A natural resource to be protected by a Pigouvian tax ? Preventive Veterinary Medicine, $\mathrm{n}^{\circ}$ 96, pp. 9-18.

Wang H., McEntire J. C., Zhang L., Li X., Doyle M. (2012). The transfer of antibiotic resistance from food to humans: facts, implications and future directions. Revue Scientifique et Technique, $\mathrm{n}^{\circ} 31$, pp. 249-260. 